\title{
10,000-fold Effect by a Nitric Oxide Donor (Sodium Nitroprusside) in Duchenne Muscular Dystrophy (DMD) Via Intrathecal Superfusion and Oral Tadalafil - A Case Report
}

\author{
Vinod Kumar Tewari ${ }^{1 *}$, Neeraj ${ }^{2}$, Devesh Johari ${ }^{3}$ and Lori Tewari ${ }^{4}$ \\ ${ }^{1}$ Director, Advance Neuro and General Hospital, Arjunaganj, Lucknow, UP, India \\ ${ }^{2}$ Physiotherapist, Advance Neuro and General Hospital, Arjunaganj, Lucknow, UP, \\ India \\ ${ }^{3}$ Ophthalmologist, Advance Neuro and General Hospital, Arjunaganj, Lucknow, UP, \\ India \\ ${ }^{4}$ Advance Neuro and General Hospital, Arjunaganj, Lucknow, UP, India \\ *Corresponding Author: Vinod Kumar Tewari, Director, Advance Neuro and \\ General Hospital, Arjunaganj, Lucknow, UP, India.
}

Received: August 25, 2020

Published: August 31, 2020

(C) All rights are reserved by Vinod Kumar

Tewari., et al.

\begin{abstract}
Duchenne Muscular Dystrophy (DMD) is a slow, progressive and fatal X-linked neuromuscular disorder due to loss of dystrophin that links the contractile apparatus to the sarcolemma via the dystrophin-associated protein complex (DPC).

The NOD (Nitric Oxide Donor) causes release of Nitric Oxide (NO) by nitric oxide synthase (NOS) and then via 10,000-fold effect NOR (Nitric Oxide Receptor) it acts to release cGMP thus modulates DPC and improves the skeleton muscle functionality.

We have used intrathecal sodium nitroprusside to activate the 10000 -fold effect to modulate the retrograde neuroregulation in DMD (diagnosed by muscle biopsy) and oral Tadalafil as NODs and well checked by AL-TENS (acupuncture like transepidermal neural stimulation) in pre ITSNP and post ITSNP phase.
\end{abstract}

Keywords: Duchenne Muscular Dystrophy (DMD); 10000-fold Effect; Intrathecal Sodium Nitroprusside; Oral Tadalafil

\section{Introduction}

Duchenne Muscular Dystrophy (DMD) is a slow, progressive and fatal X-linked neuromuscular disorder due to loss of dystrophin that links the contractile apparatus to the sarcolemma via the dystrophin-associated protein complex (DPC). If the DPC is defective then the contractile apparatus of muscle is damaged via excessive inward flow of calcium from extracellular compartment which leads to loss of regeneration and thus fibrosis of muscle fibres [1].

With the various modalities one can modulate the DPC and get better results. Like Restore NO bioavailability in dystrophic muscle including nNOS overexpression, L-arginine administration, Phosphodiesterase (PDE) inhibition and nitrate supplementation, with a focus on the effects on the architecture, function and metabolism of dystrophin-deficient skeletal muscle [1].
The nNOS (neuronal NITRIC OXIDE SYNTHETASE) is being released at the postsynaptic membrane at neuromuscular junction after activated by NMDA receptor and this in turn releases NO (NITRIC OXIDE) in the synaptic cleft which is being taken up by NOR (NITRIC OXIDE RECEPTOR). This NO donors (NODs), like Sodium nitroprusside (SNP), modulates the antegrade neurotransmission via retrograde neuroregulation by 10000 -fold effect, is well established by the previous authors [2].

In Duchenne Muscular Dystrophy (DMD) the skeleton muscle is fibrosed due to defective DPC [1]. With the more bioavailability of NO by NODs like SNP via inducing nNOS at presynaptic membrane at neuromuscular junction, the DPC acts fast. The SNP causes release of NOS and then NO causes 10000-fold effect which modulates the anterograde neurotransmission (ANT) via retrograde neurotransmission (RNT). Previous authors also postulated the negative effect 
10,000-fold Effect by a Nitric Oxide Donor (Sodium Nitroprusside) in Duchenne Muscular Dystrophy (DMD) Via Intrathecal Superfusion and Oral Tadalafil - A Case Report

of NO but those authors had skipped the fact that the superoxide dismutase (SOD) and nNOS remains active at synaptic cleft for just 5 to 7 days [3]. Up to now 2 studies have been done in humans where Tadalafil has been used but without ITSNP $[4,5]$.

We have utilised this intrathecal sodium nitroprusside (ITSNP) to induce 10000 -fold effect after $5^{\text {th }}$ day of DMD diagnosis (diagnosed by muscle biopsy) and after skipping the effect of SOD and nNOS and oral Tadalafil also for maintenance.

To quantify the effect, we have utilised AL-TENS (acupuncture like trans epidermal neural stimulation) in pre ITSNP and post ITSNP phase [6].

\section{Case Report}

A 22-year-old male presented in normal sensorium in OPD room walking with lurching gate condition with chief complaints of walking with difficulty and difficulty to stand up from sitting for 2 years with upper limbs normal. No history of tuberculosis or diabetes. On examination he has full GCS E54V5M6 (GLASGOW COMA SCALE), cranial nerves examination revealed normal 3, 4, 5, 6, 7, 8,
9 and $10^{\text {th }}$ nerve. Motor examination done with ASIA grading done in motor, sensory and bladder bowel involvement. Motor, normal nutrition of upper limbs but lower limbs both sides having moderate wasting of muscles, tone normal on both sides below C5 myotomes, power $5 / 5$ bilateral below C5 myotomes, grip 100\% on both sides. All Deep Tendon Reflexes were normal below C5. Superficial reflexes normal below $\mathrm{C} 5$ and lower limb showing normal reflexes but hypotonic gluteal muscles. The patient was asked to stand up from sitting posture then he was climbing on himself. Without any respiratory distress with single breadth count up to 49. Sensory examination is showing 224/224 (all over body normal) without bladder bowel involvement.

MRI of cervical, thoracic and lumbosacral spine done which shows normal spinal cord with thecal sac and cauda equina.

Muscle biopsy done which reported as Duchenne Muscular Dystrophy.

AL-TENS has been done which showed NORMAL on both lower limbs (figure 1, pre ITSNP, figure 2 = 15 days POST ITSNP).

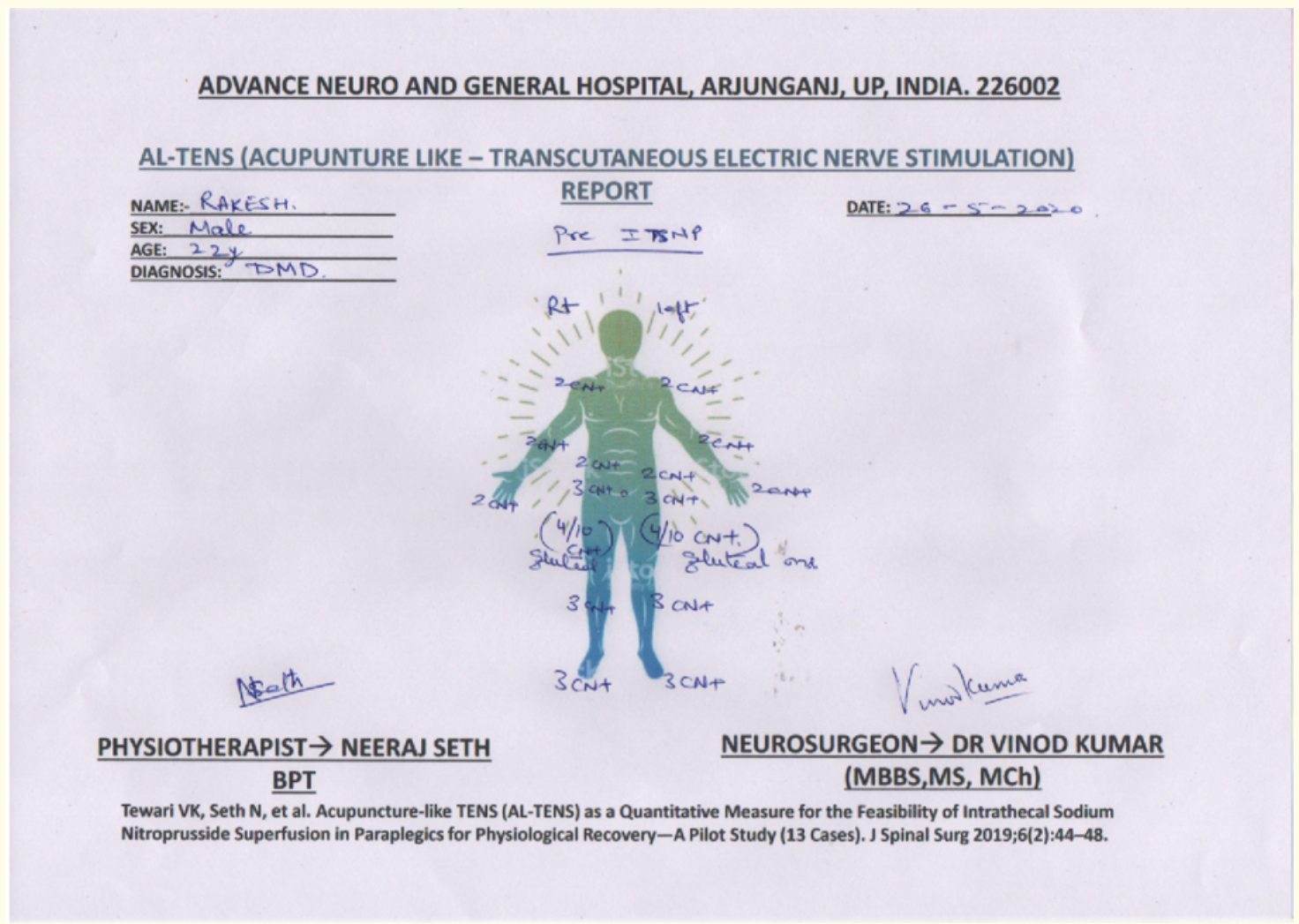

Figure 1: Pre ITSNP ALTENS. 


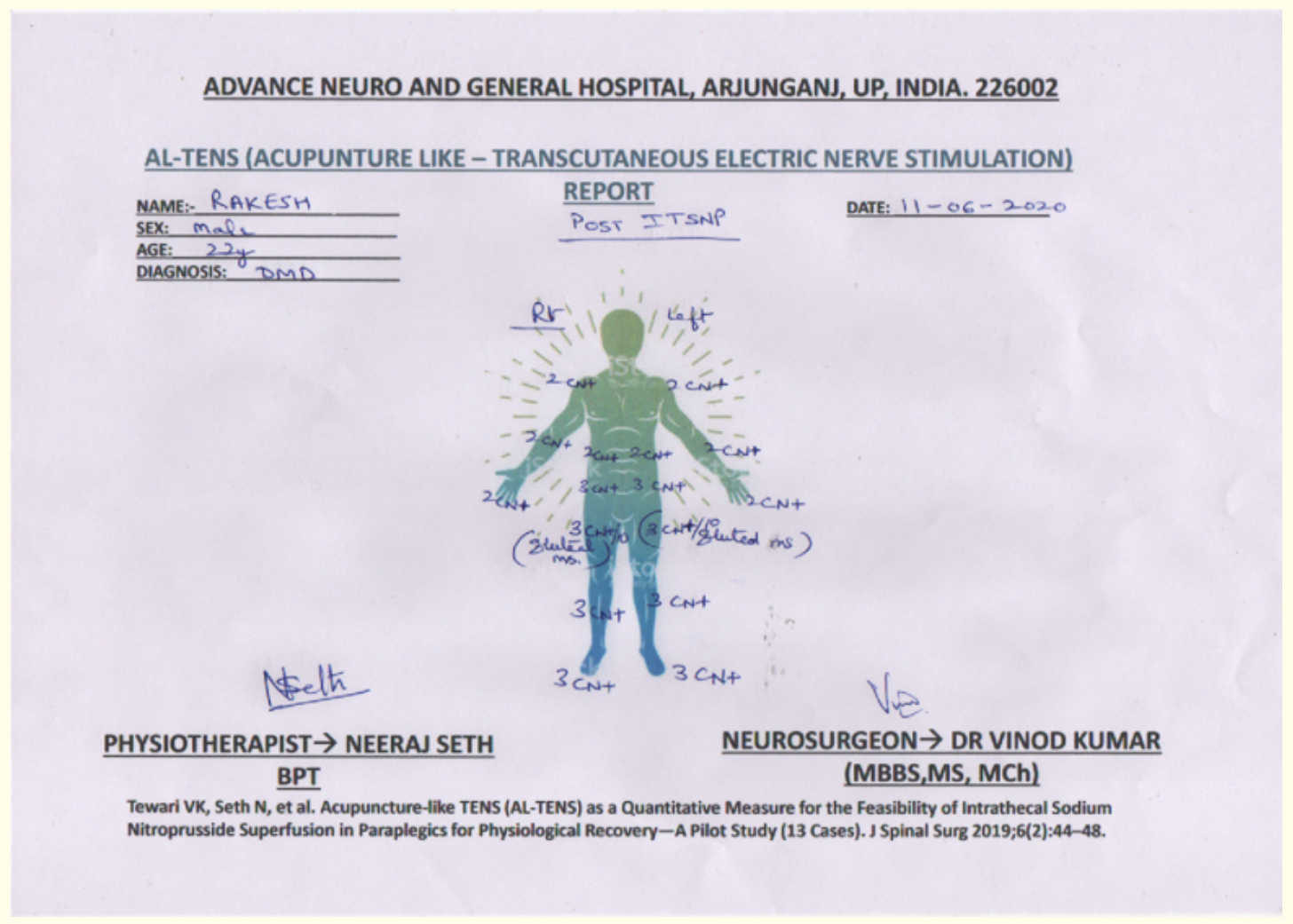

Figure 2: Post ITSNP $15^{\text {th }}$ day ALTENS.

After taking written confirmation that the patient in the study has given consent for the video recording and its clinical study to be published and well informed consent about all untoward action (like sweating and apprehension) of ITSNP we superfused ITSNP about $15 \mathrm{ml}$ of the SNP given of $50 \mathrm{mg}$ of SNP dissolved in 200 $\mathrm{ml}$ of Dextrose 5\% solution with full photoprotection and freshly prepared. Post ITSNP AL-TENS done again after 7 days and $14^{\text {th }}$ day and video recordings done also. Then after ITSNP patient was given ORAL TADALAFIL $20 \mathrm{mg} 1$ TDS for next 7 days.

YouTube URL of PRE ITSNP and POST ITSNP phase is:

Video 1- Pre ITSNP phase.

Typical of DMD

Video 2- Post ITSNP 7 days

https://youtu.be/0HRab6XLDuc

https://youtu.be/bg2vpQWNK7s

Video 2- Post ITSNP 2 months

https://youtu.be/uN9m0jWtoSc

https://youtu.be/XbaXBfDi8P0

\section{Discussion}

The DMD is a dreadful slow progressive disease. Mostly affects due to defective $\mathrm{DPC}^{2}$ mechanism. The NOD releases NO which causes modulation of ANT via RNT by 10000 -fold effect, thus increases the ANT impulses in those defective DPC by bypassing the routine ANT impulses [1-3].

After cGMP activation via NOR, cGMP activates DPC and then the contractile apparatus causes appropriate contraction of muscle as such. With the deficient DPC, excessive intake of calcium is seen inside the sarcolemma and thus muscle's regeneration is hampered with excessive damage and fibrosis. With the increased nNOS activation via NOD the DPC gets optimal activation instead of low frequency, so the muscle contraction is benefitted with each anterograde neurotransmission.

As far as human's use of TAB TADALIS is concerned it has been used in humans in two studies and both reported good recovery, we have used oral Tab TADALIS along with ITSNP so that the 10000fold effect will cause swift modulation and then oral TAB TADALIS will maintain the effect later on. And got an excellent result. 
The previous studies has been based on the muscle ischemia studies but we have utilised AL-TENS to quantify the neuromuscular junction functionality.

From our work [3] it was well proved that the SOD level and nNOS level comes to normal after 5 to 7 days and if we skip this time the 10000-fold effect comes to action to generate the ANT via RNT well tested via AL-TENS $[5,6]$.

\section{Conclusion}

This case was well diagnosed as DMD and after giving ITSNP to induce the 10000 -fold effect got $35 \%$ improvement on $15^{\text {th }}$ day of post ITSNP phase.

\section{Bibliography}

1. Cara A Timpani., et al. "Therapeutic strategies to address neuronal nitric oxide synthase deficiency and the loss of nitric oxide bioavailability in Duchenne Muscular Dystrophy". Orphanet Journal of Rare Diseases 12 (2017): 100.

2. Tewari VK., et al. "The 10,000-Fold-Effect-Retrograde Neurotransmission- A Newer Concept for Paraplegias Physiological Revival-Use of Intrathecal Sodium Nitroprusside". Journal of Evolution of Medical and Dental Sciences 3.26 (2014): 72707285.

3. Vinod Kumar Tewari., et al. "Intracarotid Sodium Nitroprusside on Fifth Post Ischemic Stroke Day in Middle Cerebral Artery Occlusion Rat Model". Journal of Clinical and Diagnostic Research 11.8 (2017): AF01-AF04.

4. Nelson MD., et al. "PDE5 inhibition alleviates functional muscle ischemia in boys with Duchenne muscular dystrophy". Neurology 82 (2014) :2085-2091.

5. Martin EA., et al. "Tadalafil alleviates muscle ischemia in patients with Becker muscular dystrophy". Science Translational Medicine 4 (2012): 162ra155.

6. Tewari VK., et al. "Acupuncture-like TENS (AL-TENS) as a Quantitative Measure for the Feasibility of Intrathecal Sodium Nitroprusside Superfusion in Paraplegics for Physiological Recovery-A Pilot Study (13 Cases)". Journal of Spine Surgery 6.2 (2019): 44-48.

\section{Assets from publication with us}

- Prompt Acknowledgement after receiving the article

- Thorough Double blinded peer review

- Rapid Publication

- Issue of Publication Certificate

- High visibility of your Published work

Website: www.actascientific.com/

Submit Article: www.actascientific.com/submission.php

Email us: editor@actascientific.com

Contact us: +919182824667 